\title{
A Simple and Low-Cost, but Naturalistic Method for Introducing Students to Action Potentials
}

\author{
Orlando Jorquera ${ }^{1^{*}}$ \\ https://orcid.org/0000-0003-4086-5474
}

\section{Edgar Harry Vogel ${ }^{2}$}

https://orcid.org/0000-0001-6999-6991

${ }^{1}$ Federal University of Southern Bahia- UFSB, Porto Seguro, Bahia, Brazil; ${ }^{2}$ University of Talca, Talca, Chile.

Editor-in-Chief:Paulo Vitor Farago

Associate Editor: Paulo Vitor Farago

Received: 2020.04.24; Accepted: 2020.12.18.

*Correspondence: ojorquerc@gmail.com; Tel.: +55-73-3288-8400. (O. J.).

\section{HIGHLIGHTS}

- Action potential (AP) in earthworm.

- Low-cost amplifier for AP determination.

- Big neural cord in earthworm.

- Introducing students to neuroscience.

\begin{abstract}
Current trends in science education recommend the complementary use of virtual and hands-on methods of teaching. In neurobiology, for instance, there is a plethora of virtual laboratories and simulators that can be readily combined with traditional physical labs. Unfortunately, physical laboratories are almost unaffordable for many institutions due to the high cost of equipment. In this paper, we present a simple and low-cost in vivo method for demonstrating some of the basic biophysical properties of neural action potentials. The method involves the following steps: a) dissection of the ventral nerve cord of earthworm; b) electrical stimulation; c) amplification and visualization of the medial and lateral giant fibers' action potentials; and d) recording. The system showed stability, expected amplification, high signal-to-noise ratio, and an estimated total cost of US $\$ 5.662$. We provide guidelines for assembling the system and discus its utility as a teaching alternative for low budget institutions.
\end{abstract}

Keywords: Action potential; low-cost action potential laboratory; neurobiology lab; equitable science education.

\section{INTRODUCTION}

There has been a long-lasting consensus among science educators that practical laboratory activities are an essential part of teaching and learning of any discipline [1-7]. Among other things, laboratories, or more generally said, "hands-on activities", allow for linking two of the most fundamental components of science: theory and experimentation. Moreover, it has been shown that early involvement in hands-on research encourages students to pursue studies in science [8] and to reduce the achievement gap of disadvantageous learners [9]. 
Regrettably, in many circumstances financial issues impose limits in the design and implementation of laboratories. In several areas of basic science, the equipment, facilities, and supplies that would be necessary to provide students with a significant learning experience are far above institutional budgets. These constraints have been partially mitigated by using virtual laboratories and simulations [10,11]. Although there has been some debate [12] authors seem to have reached consensus that both, virtual and physical laboratories, have relative strengths and weaknesses and they should be optimally used in combination with each other [13-14].

Basic neurobiology is not an exception in this panorama. Consider, for instance, a simple in vivo demonstration of an action potential. The standard setup for this type of demonstration comprises several items, such as micromanipulator, microscope, stimulator, oscilloscope, amplifier, and data acquisition software, whose total cost is around US $\$ 15,000$. As an alternative, some open-source online simulators of action potentials have been made available by researchers (see for instance, https://phet.colorado.edu/sims/html/neuron/latest/neuron_en.html and http://nerve.bsd.uchicago.edu provided by the universities of Colorado and Chicago, respectively).

Although simulators have a demonstrated utility as a teaching tool in biology [15-17], many educators believe that they cannot replace the vivid, active and holistic experience of laboratory demonstrations (NRC 2006, NSTA, 2007).

Consequently, there have been some attempts to design low-cost in vivo procedures for demonstrating neuronal action potentials [19-23]. For instance Kladt and coauthors [20] and Shannon and coauthors [22] reported step by step lab demonstrations of action potentials in the earthworm. Unfortunately, the report by Kladt and coauthors lacks the necessary details for implementation and the method proposed by Shannon and coauthors produced records of poor quality. Furthermore, both studies describe methods to display biphasic action potentials, which is not what the students normally see in textbooks. Despite these problems, the use of earthworms for didactic demonstrations of action potential has number of advantages, such as the ubiquity of the animal, easiness of manipulation and availability of information on its neural structures. In this paper, we describe an alternative approach for demonstrating action potentials in earth worm which is even less expensive than its predecessors, has better signal-to-noise ratio and displays monophasic signals. We believe that these features complement previous efforts and improve the relationship between cost and quality of recording.

\section{MATERIAL AND METHODS}

The procedure was an adaptation of that described by Roberts (1962) [24] and it is sketched in Figure 1. Earthworms of the species Lombricus terrestris were obtained around the Sosígenes Costa Campus of the Federal University of Southern Bahia, Porto Seguro, Bahia, Brazil. For maintenance, they were placed in a container filled with soil and minced banana. The ambient temperature was kept constant at $28{ }^{\circ} \mathrm{C}$ throughout the experiment. 

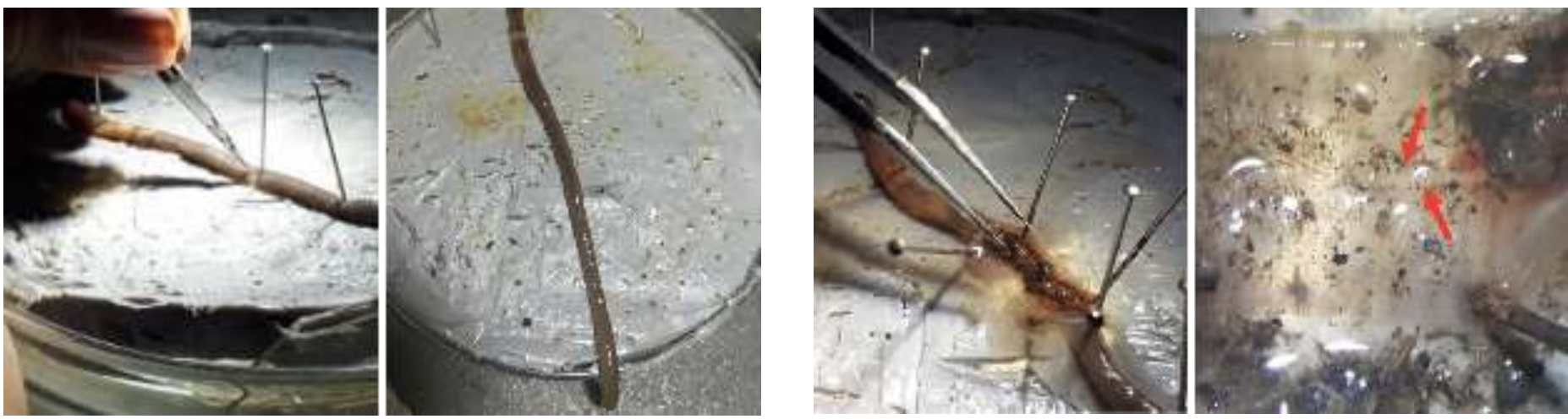

(a)

(b)
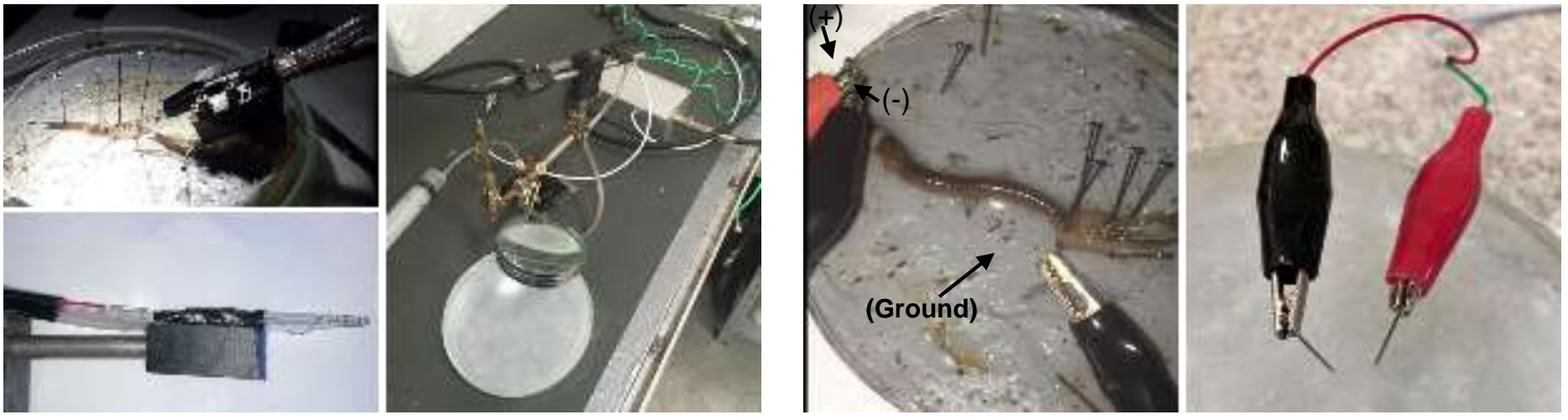

(c)

(d)

Figure 1. Experimental set up for recording action potentials from a ventral neural cord in the earthworm. See the text for more details.

$(-)$

The animal was then anesthetized using $10 \%$ alcohol tor 6 minutes, washed with water to remove alcohol residue, and placed in a petri dish filled with hardened silicone with the dorsal side up and pinned to $2 / 3$ of the cephalic part (Figure 1a). Next, using a low-cost common magnifying glass to amplify the tissue, the dorsal part was cut with scissors and fixed with pins being careful not to damage the intestine and nerves (Figure 1b). The digestive system was then removed with tweezers to expose the ventral nerve cord. In order to remove remaining tissue and other debris and to maintain adequate salinity for optimal recording, we added a saline solution containing $\mathrm{NaCl}(6 \mathrm{~g} / \mathrm{L}), \mathrm{KCl}(0.12 \mathrm{~g} / \mathrm{L}), \mathrm{CaCl}_{2}(0.2 \mathrm{~g} / \mathrm{L})$ and $\mathrm{NaHCO}_{3}(0.1 \mathrm{~g} / \mathrm{L})$. The nerve was then cut at the bottom with scissors and separated from the tissues by using the tip of a suction electrode.

The loose nerve was suctioned with a glass suction electrode consisting of an internal $\mathrm{Ag} / \mathrm{AgCl}$ electrode and an $\mathrm{Ag} / \mathrm{AgCl}$ outer coiled to a $1-\mathrm{mL}$ pipette tip, attached to a $30 \mathrm{~cm}$ tube and a $5 \mathrm{~mL}$ syringe similar to that described by Johnson and coauthors [25]. To chloritizing the silver wire, it was placed overnight in a 5\% solution of sodium hypochlorite. Instead of attaching the electrode to a micromanipulator, as it is commonly done, it was attached to a low- cost manipulation system consisting of articulated metal bars and connected to the amplifier (Figure 1c).

The electrical stimulus was delivered thought two iron pins. The cathode (+) and the anode (-) were inserted consecutively in the cephalic part of the earthworm separated by approximately $5 \mathrm{~mm}$. A third electrode was placed between the first pair of electrodes and the nerve cord and served as ground (Figure 1d). Action potentials were amplified and visualized using a Tektronix TBS 1072B digital oscilloscope. To reduce electrical noise, the computer was run on battery power and the amplifier was placed inside a faraday 
cage. To determine the conduction velocity $(C V)$, the following equation was used: $C V=d / l a t e n c y$, where $d$ is the distance between the stimulation electrodes and the suction electrode $(5 \mathrm{~cm}$ approximately) and latency is the time between stimulation and the first deflection from baseline.

The amplification system comprises two follower amplifiers connected to the input of a differential amplifier (INA128P) (http://ti.com/lit/ds/symlink/ina128.pdf) and to an offset regulator (made with an amplifier type TL071CN, http://ti.com/lit/ds/symlink/tl0). The internal electrode of the suction electrode was connected to one follower amplifier whose output is supplied to the non-inverting input of the differential amplifier. In turn, the external electrode (rolled up into the suction electrode) was connected to the other follower amplifier whose output is supplied to the inverting input of the differential amplifier. The output of the differential amplifier was connected to the inverted offset regulator which produces finally a non-inverted signal for the inverting input and an inverted signal for non-inverting input. Thus, the system first detects the potential variation in the non-inverting input (internal suction electrode) compared to the inverting input (external suction electrode) which provides the expected response to an extracellular register. Furthermore, the offset regulator allows for adjusting the baseline close to $0 \mathrm{mV}$. The circuit was powered by two 9-volt batteries and it is sketched in Figure 2.

INA $128 p$

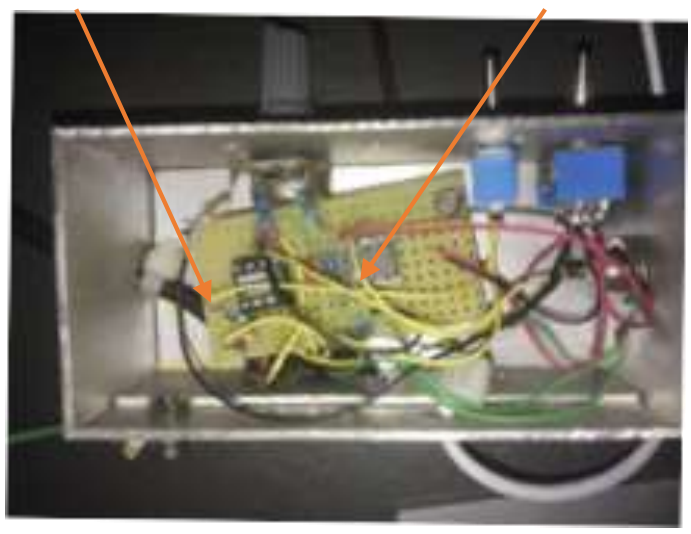

(a)

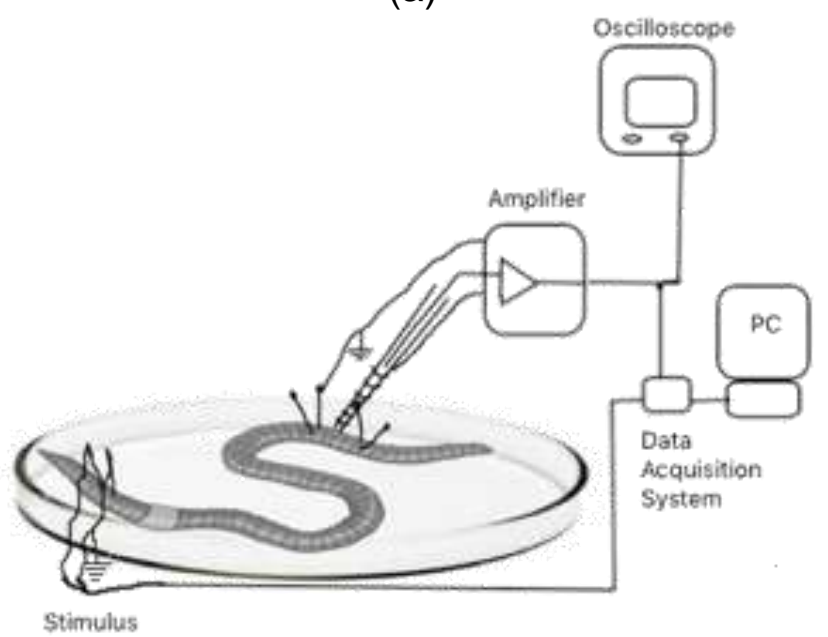

(b)

(c)

Figure 2. Low-cost amplifier for recording action potentials. a) Photograph of the assembled system; b) Assembled circuit comprising two low power instrumentation amplifiers (INA128p and TL071CN) and one offset regulator. c) experimental system diagram indicating the animal used the stimulation, amplification, visualization (oscilloscope) and acquisition and recording system (USB-6218, computer).

The gain of the amplifier was 99 and 1065 times calculated as $\mathrm{G}=1+50 \mathrm{kohms} / \mathrm{Rg}$, where $\mathrm{Rg}$ is the external resistance to be modified with 511 ohms and 47 ohms to have the gains described. 
The signal-to-noise ratio was calculated as $S N R=20 \log 10\left(A_{\text {signal }} / A_{\text {noise }}\right)$, where $A_{\text {signal }}$ and $A_{\text {noise }}$ are the respective root mean square. The amplification system and the data acquisition hardware were placed inside a Faraday cage connected to ground. Data acquisition and electrical stimulation were performed through a USB-6218 digital-analog converter with $250 \mathrm{KS} / \mathrm{s}$ (kilo-samples by second) controlled by Labview software (National Instruments). This permitted the administration of $100 \mu$ s pulses with amplitude ranging from $0-10$ Volts at 1-s intervals. The rate acquisition was at $50 \mathrm{kHz}$ and stored in .Ivm format (Labview measurement) and finally imported and analyzed using the Excel program.

\section{RESULTS}

The amplification circuit was assembled as described in materials and methods. It exhibited a very stable response and high signal to noise ratio of $25 \mathrm{~dB}$, which allowed not to use a filter. The total cost of the amplification system was approximately US\$24.8 (see Table 1), having a much lower cost than any other commercial system designed for the same purpose. The most expensive items were the oscilloscope (US\$ 800), the LabView program (US\$ 2990) and the digital-analog converter USB-6218 (US\$1618), all of which can be used for additional purposes. Thus, although the proposed system considerably reduced the cost of amplification did not avoid the use of high-cost equipment such as an oscilloscope and a data acquisition system. The stimulation system has been simplified using the same acquisition system, but this stimulus can also be generated using other platforms such as Arduino with limitation of the output voltage of the equipment to 5 volts.

Table 1. Material needed to build the amplifier circuit and associated cost. Value quoted in Digi-key electronics (www.digikey.com), Mouser electronics (www.mouser.com) and National Instruments (www.ni.com).

\begin{tabular}{lccc}
\hline Component & Quantity & Cost US\$ & Total (US\$) \\
\hline INA128p & 1 & 11.16 & 11.06 \\
TL071 & 1 & 0.4 & 0.4 \\
Resistor 511 ohm & 1 & 0.11 & 0.11 \\
Resistor 47 ohm & 1 & 0.1 & 0.1 \\
Resistor 1kohm & 1 & 0.12 & 0.12 \\
Resistor 715 ohm & 3 & 0.18 & 0.54 \\
Capacitor 0.1 uF & 2 & 0.23 & 0.46 \\
Aluminum case 5x10x4 cm & 1 & 8.45 & 8.45 \\
Switch & 2 & 0.87 & 1.74 \\
Potentiometer 10K & 1 & 1.83 & 1.83 \\
Subtotal (US\$) & & & 24.81 \\
Low-cost magnifying glass and a manipulator & 1 & 1 & 7.97 \\
Oscilloscope Tektronix TBS1072B & 1 & 863 & 863 \\
USB-6218 & 1 & 1618 & 1618 \\
Labview & 1 & 3149 & 3149 \\
Subtotal (US\$) & & & 5637.97 \\
Total (US\$) & & & 5662.8 \\
\hline
\end{tabular}

Figure 3 illustrates the kind of signal that can be obtained with our device. After suctioning the nerve with the suction electrode and turning on the amplifier, it is possible to observe spontaneous fluctuations of potential (Figure 3A). These records are very reproducible if the earthworm is slightly anesthetized $(<6 \mathrm{~min})$ and the nerve cord has been properly suctioned. The inner diameter of the pipette must be similar to the nerve diameter, which allows to increase the resistance between the internal and external $\mathrm{Ag} / \mathrm{AgCl}$ electrodes and to observe the variation of the electrical potential. 


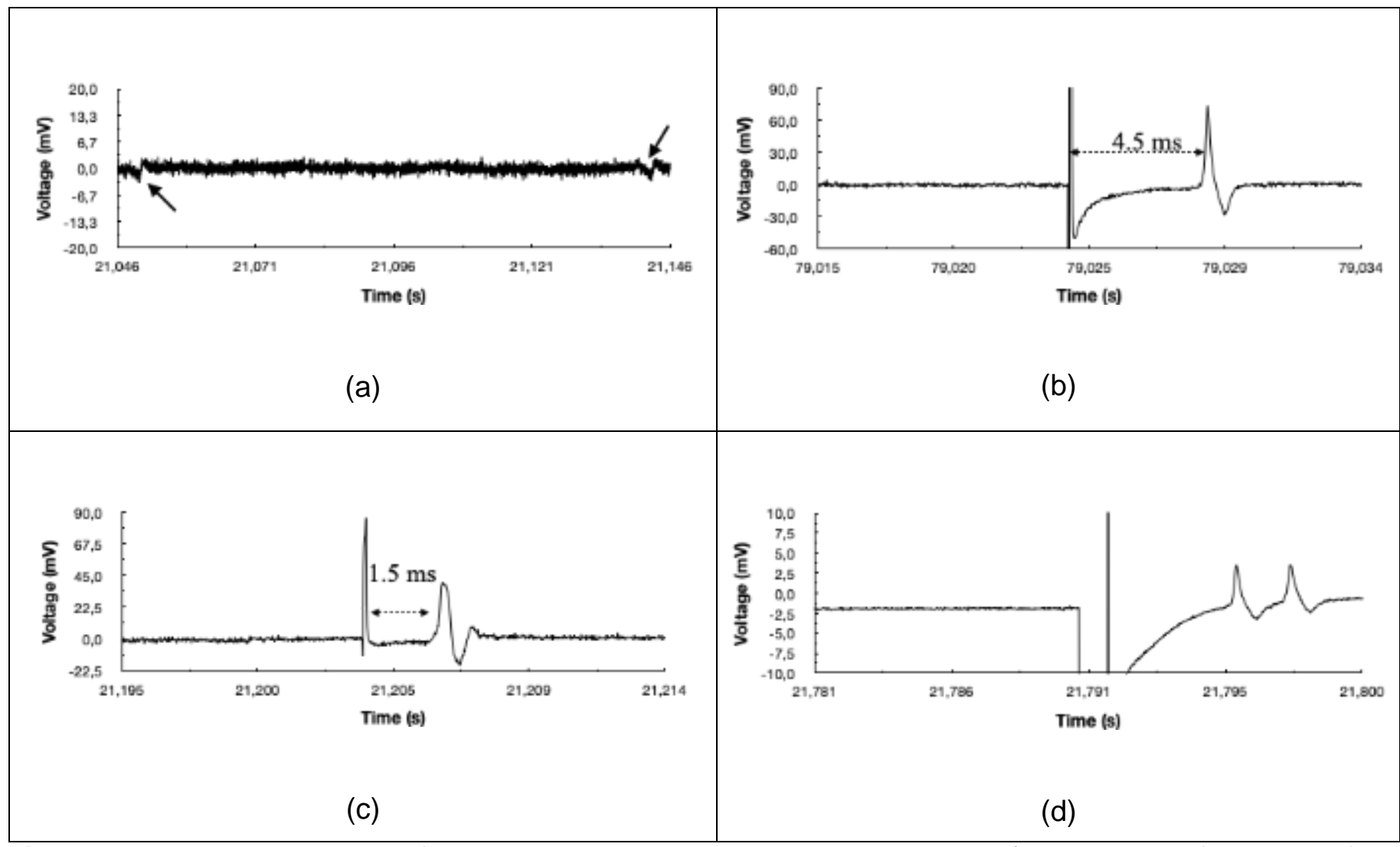

Figure 3. Action potential record from a ventral neural cord in the earth- worm. A) spontaneous fluctuation of AP (indicated by continuous arrow) without electrical stimulus; B) characteristic AP lateral giant fiber with a latency of 4.5 $\mathrm{ms}$; C) characteristic AP medium giant fiber with latency of $1.5 \mathrm{~ms}$; D) Double AP by increased the stimulus duration from 100 us to $1 \mathrm{~ms}$ indicating the activation of both fibers in the nerve cord.

Under electrical stimulation conditions, there are characteristic fluctuations in the signal revealing the development of an action potential, with a pronounced depolarizing event followed by repolarization. It was possible to record action potentials from the lateral and medial giant fibers, whose conduction time was approximately $4.5 \mathrm{~ms}$ and $1.5 \mathrm{~ms}$, respectively (Figures $3 \mathrm{~b}$ and $3 \mathrm{c}$ ). This difference is due to the fact that increasing the diameter of the axon, increase their conduction velocity in a relationship that is the square root of the axon diameter; [22,26]. The diameter of the giant fiber is $0.05 \mathrm{~mm}$ for the LGF and $0.07 \mathrm{~mm}$ for the MGF approximately.

The peak-to-peak amplitude was approximately $100 \mathrm{mV}$ (Gain =100) for LGF (Figure 3B) and $60 \mathrm{mV}$ for MGF (Figure 3C). Since the measured value is given by the potential drop in external resistance, these values depend on the resistance between the reference and recording electrodes in the suction pipette. The conduction velocity was $11 \mathrm{~m} / \mathrm{s}$ for LGF and $33 \mathrm{~m} / \mathrm{s}$ for MGF. These values are similar to those found by several authors [20,22,26,27]. According to Shannon and coauthors [22], a difference of 1.4 times the conduction velocity between LGF and MGF is expected, but it depends on the fiber size, which may vary depending on the earthworm being analyzed. Doubles AP were also observed when the ventral nerve cord was stimulated with a greater potential (from 1 to $9 \mathrm{~V}$ ) or when the duration of the pulse increased from 100 $\mu$ s to $1 \mathrm{~ms}$ (Figure 3D). This is because the stimulation voltage or pulse duration is high enough to stimulate both fibers in the nerve cord (MGF and LGF).

\section{DISCUSSION}

There are several potential problems that might arise during stimulation and recording that require a careful selection of materials and adequate preparation of the animal. First, if the earthworm is anesthetized for more than 6 minutes, it is very likely that not spike will be observed (a situation similar to that described by Shannon and coauthors [22]). Second, since AP logging is often out of baseline, the use of the offset regulator is essential. Third, the pipette tip of the suction electrode must have a diameter similar or smaller than that of the neural cord, otherwise AP may not be observed. Four, failure to adhere properly greatly decreases the resistance between the electrodes and an AP might not be observed. A system of articulated metal bars was used as a guide to the suction electrode, but a good approximation of the electrode to the 
neural cord is difficult. The use of a micro-manipulator system is very advisable to increase reliability of the procedure, but it is expensive.

Over the past decade, there have been a few reports of successful use of low-cost equipment for class demonstrations of action potential in earth- worms [20,22,23,28]. Although some of these studies did not achieve good SNR (e.g., [22]), and none describe how the amplification system can be built, as we do in the present article, they are very valuable because they provided a set of experiments that can be conducted in the classroom. The present work adds to these efforts by providing a detailed description of how to build a stimulation/amplification system for this purpose.

In sum, in this paper we describe a method to build a very low-cost amplifier manufactured with parts that can be found in any electronics store. The system has an excellent SNR when placed in a faraday cage and powered with batteries. The handling and stimulation procedures involve common laboratory supplies, such as a $1 \mathrm{ml}$ pipette tip and chlorinated silver wires with sodium hypochlorite. No micro manipulator is required. The system can be built by students without great knowledge in electronics.

We believe that this sort of tools may stimulate student's curiosity for basic processes in neurophysiology and we hope that it will encourage teachers to consider other low-cost educational strategies in related domains, such as voltage-clamp or current analysis.

Acknowledgments: We thank Professor Francisco Bezanilla Ph.D. for his valuable help and advice in assembling the circuit and experimental system. This work was partially supported by PIA Ciencia Cognitiva, Centro de Investigación en Ciencias Cognitivas, Facultad de Psicología, Universidad de Talca.

Conflicts of Interest: The authors declare no conflict of interest. The funders had no role in the design of the study; in the collection, analyses, or interpretation of data; in the writing of the manuscript, or in the decision to publish the results.

\section{REFERENCES}

1. National Research Council (NRC). America's lab report: Investigations in high school science. Washington, DC: National Academy Press. 2006.

2. Hofstein A, Lunetta VN. The role of the laboratory in science teaching: Neglected aspects of research. Rev Educ Res.1982;52(2):201-17.

3. Hoftein A, Lunetta VN. The laboratory in science education: Foundations for the twenty-first century, Sci Educ.2004;88(1):28-54.

4. Lazarowitz R, Tamir P. Research on using laboratory instruction in science. In D. Gabel editors. Handbook of research on science teaching and learning. New York. The Macmillan Publishing Company. 1994;94-130.

5. Hofstein A, Mamlok-Naaman R. The laboratory in science education: the state of the art. Chem. Educ. Res. Pract. 2007;8(2):105-7.

6. Lunetta VN, A. Hofstein, A. Learning and teaching in the school science laboratory: An analysis of research, theory, and practice. I.n N. Leaderman and S. Abel editors. Handbook of research on science education. New York. Lawrence Eralbaum. 2007;393-441.

7. Stohr-Hunt PM. An analysis of frequency of hands-on experience and science achievement. Journal of Research in Science Teaching: The Official Journal of the National Association for Research in Science Teaching. 1996;33(1):101-9.

8. Russell SH, Hancock MP, McCullough J. Benefits of undergraduate research experiences. Science. 2007;316 (5824): 548-9.

9. Haak, DC, HilleRisLambers J, Pitre E, Freeman S. Increased structure and active learning reduce the achievement gap in introductory biology. Science, 2011;332:1213-16.

10. National Research Council (NRC). Learning science through computer games and simulations. Honey MA and Hilton ML Eds., Board on Science Education. Washington DC.The National Academies Press;2011.

11. Scanlon E, Colwell C, Cooper M, Di Paolo T. Remote experiments, re-versioning and re-thinking science learning. Comput Educ.2004;43(1-2):153-63.

12. Ma J, Nickerson JV. Hands-on, simulated, and remote laboratories: A comparative literature review. ACM Comput Surv (CSUR).2006;38 (3):1-24.

13. De Jong T, Linn MC, Zacharia ZC. Physical and virtual laboratories in science and engineering education. Science. 2013;340(6130):305-8.

14. Sauter M, Uttal DH, Rapp DN, Downing M, Jona K. Getting real: the authenticity of remote labs and simulations for science learning. Distance Educ.2013;34(1):37-47. 
15. Tsihouridis $C$, Vavougios $D$, Batsila $M$, loannidis $G$. The optimum equilibrium when using experiments in teachingwhere virtual and real labs stand in science and engineering teaching practice. Int. J. Emerg. Technol. Learn (iJET).2019;14(23):67-84.

16. Son JY. Comparing physical, virtual, and hybrid flipped labs for general education biology. Online Learning. 2016;20(3):228-43.

17. Develaki M. Methodology and epistemology of computer simulations and implications for science education. J Sci Educ Technol.2019;28(4):353-70.

18. Lai JW, Bower M. Evaluation of technology use in education: Findings from a critical analysis of systematic literature reviews. J Comput Assist Learn.2019;1-19.

19. Dagda RK, Thalhauser RM, Dagda R, Marzullo TC, Gage GJ. Using crickets to introduce neurophysiology to early undergraduate students. J. Undergrad. Neurosci. Educ.2013;12(1):A66-A74.

20. Kladt N, Hanslik U, Heinzel HG. Teaching basic neurophysiology using intact earthworms. J. Undergrad. Neurosci. Educ.2010;9(1):A20-A35.

21. Marzullo TC, Gage GJ. The spikerbox: a low cost, open-source bioamplifier for increasing public participation in neuroscience inquiry. PloS one. 2012;7(3):1-6.

22. Shannon KM, Gage GJ, Jankovic A, Wilson WJ, Marzullo TC. Portable conduction velocity experiments using earthworms for the college and high school neuroscience teaching laboratory. Adv Physiol Educ. 2014;38(1):6270 .

23. Drewes CD. Non-invasive recording of giant nerve fiber action potentials from freely moving oligochates. In: Tested Studies for Laboratory Teaching, edited by Karcher SJ. Chapel Hill, NC: Proceedings of the 20th Workshop/Conference of the Association for Biology Laboratory Education. vol. 20, chapt. 2; 1999. 45-62.

24. Roberts $M$. The giant fibre reflex of the earthworm, lumbricus terrestris I: I. the rapid response. J. Exp. Biol. 1962;39(2):219-27.

25. Johnson BR, Hauptman SA, Bonow RH. Construction of a simple suction electrode for extracellular recording and stimulation. J. Undergrad. Neurosci. Educ. 2007;6(1):A21.

26. Günther J. Impulse conduction in the myelinated giant fibers of the earthworm. Structure and function of the dorsal nodes in the median giant fiber. J. Comp. Neurol. 1976;168(4):505-31.

27. Rushton WAH. Action potentials from the isolated nerve cord of the earthworm. Proc. R. Soc. Lond. 1945;B 132 (869):423-37.

28. Bähring $\mathrm{R}$, Bauer CK. Easy method to examine single nerve fiber excitability and conduction parameters using intact non anesthetized earth- worms. Adv Physiol Educ. 2014;38(3):253-64.

2021 by the authors. Submitted for possible open access publication under the terms and conditions of the Creative Commons Attribution (CC BY NC) license (https://creativecommons.org/licenses/by-nc/4.0/). 